\title{
Determining Disease Load through the National Health Information System in Pakistan
}

\author{
Syed M. Mursalin* \\ National Health Institute, National Health Inforamtion/Disease Surveillance Centre, Islamabad, Pakistan
}

\section{Objective}

Purpose of this abstract is to show how launch of a standard National Health Information System is has become the main national data source, and is, playing a pivotal role in facilitating decision making in health care system in Pakistan.

\section{Introduction}

Before the launch of standard National Health Information System (NMHIS) in 2000, there had been acute paucity of reliable and timely health information in Pakistan. Health Departments had no choice than to resort to estimates or carry expensive community based surveys to determine the disease incidence. After the development and deployment of NHMIS, overall Health System is reshaping itself based upon the regular and frequent information now available on a good number number of priority health problems. This is system is now offering tremendous opportunities for promoting the cause of evidence based decision making and monitoring of its expanded health care structure. This effort had led to putting in place a standard system of data collection and transmission from roughly 13000 public health services (both urban and rural ). The new system is now able to promptly locate pockets of vulnerable communities reporting high disease incidence.

\section{Methods}

Use of Secondary data gathered through National Health Information System data collection protocols.

\section{Results}

Recently published 'National HIS Report- 2013 has made some alarming revelations This report is developed after an analysis of estimated 118 million visits made to health services during 2013 . Out of 43 reportable diseases, 22 diseases are communicable in nature. This system is providing some useful information not only to the District Health Managers, but also to national managers of Public Health Programs (eg Malaria (DOTs), EPI Tuberculosis, Hepatitis, AID.HIV, EPI.) This analysis has revealed that out of the total visits during 2013, $53 \%$ belonged to 43 priority diseases selected for HIS reporting. From this total a high percentage of patients belonged to acute respiratory infections (30\%). This was followed by gastro intestinal diseases (14.8\%), Scabies (8\%) and oral diseases (3\%). Other communicable diseases, like measles, meningitis, malaria (10\%) comprised the next group. Non communicable diseases (selected for HIS reporting) comprised around $24 \%$ of the whole patient load.

Recording for preventive services show that 3.28 million pregnant women visited health facilities for antenatal care services during the year 2012. Unfortunately, a high rate of anemic women that is around $30 \%$ of pregnant women were recorded (ie. $\mathrm{Hg}<10 \mathrm{gm} \%$ ). Whereas, 0.7 million $\mathrm{C}$ sections were reported from these facilities. System also gathers information on deaths and cause of deaths with special emphasis on maternal deaths. This system is capturing vital information on some of critical priority diseases, for which no other data collection mechanism is currently available. This include information about roadside accidents, rabies, snake bite, sexually transmitted diseases and typhoid. Similarly, NHIS is one of the main data source also for some non communicable and other diseases/ accidents including diabetes, ischemic health diseases, liver cirrhosis, epilepsy, dental cares, burns and eye diseases.

\section{Conclusions}

There is a strong justification to strengthen and promote already functional routine health information systems by putting in more investment and emphasis in its infrasructure, hards and software support, staff capacity building in data generation and use of information. At this stage of achievement it is now the time for promoting the culture of evidence based decision making specially to address its dismal disease profile.

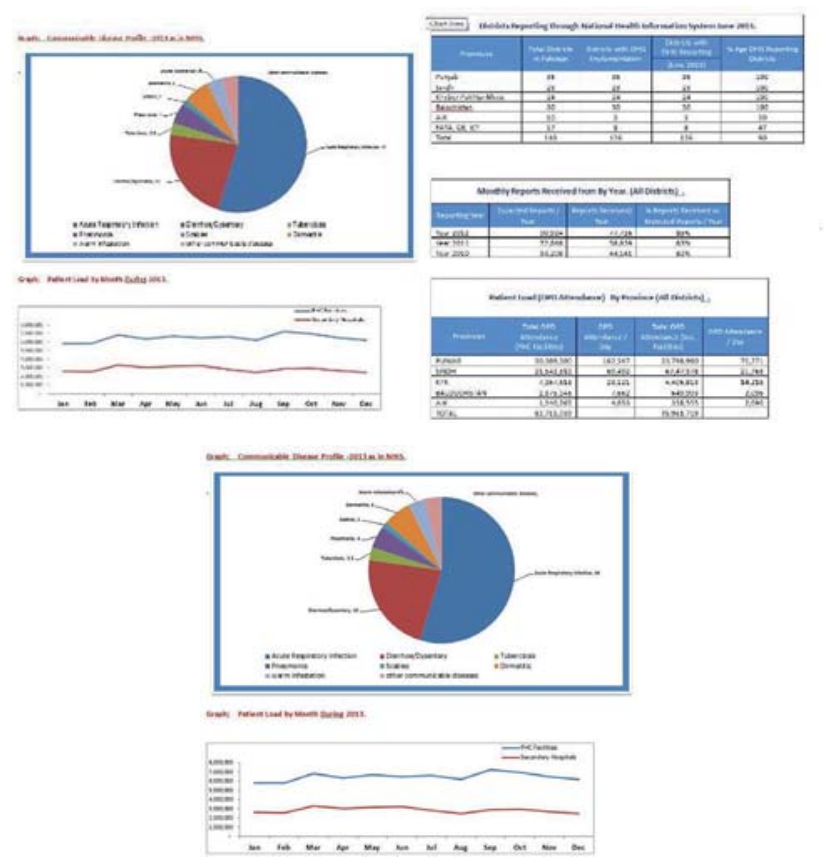

Keywords

National Health Information; Cost Effectiveness; Evidence Based Decision Making.; Standard National System.; Health Managers

\section{Acknowledgments}

Dr. Shagufta Zareen, Data Analyst/Coordinator, National Center for Health Information/Surveillance- Islamabad, Pakistan

\section{References}

National Health Information System, Ministry of Health Services, Pakistan.

\section{*Syed M. Mursalin}

E-mail: mursalin831@gmail.com 